\title{
Reviews of "Neutralization of N501Y mutant SARS- CoV-2 by BNT162b2 vaccine-elicited sera"
}

Kabir Biswas ${ }^{1}$, Kimberly Luke ${ }^{2}$, Geraldo Passos ${ }^{3}$, Binquan Luan ${ }^{4}$, Haoran Wang 5

${ }^{1}$ Hamad Bin Khalifa University, College of Health \& Life Sciences, Qatar,

2 Intuitive Biosciences, Research and Development, USA,

${ }^{3}$ Associate Professor, University of Sao Paulo School of Dentistry of Ribeirao Preto, Brazil, ${ }^{4}$ IBM T J Watson Research, Computational Biological Center, USA, ${ }^{\mathbf{5}}$ Neoland Bioscience

Published on: Feb 07, 2021

License: Creative Commons Attribution 4.0 International License (CC-BY 4.0). 
To read the original manuscript, click the link above.

Summary of Reviews: This preprint showed data that BNT162 b2 vaccine may protect against a spike mutation associated with rapidly spreading SARS-CoV-2 strains, but more work needs to be done.

Reviewer 1 (Kabir Biswas) |

Reviewer 2 (Kimberly Luke) |

Reviewer 3 (Geraldo Passos) |

Reviewer 4 (Binquan Luan, Haoran Wang) |

RR:C19 Strength of Evidence Scale Key.

$\square \square \square \square \square=$ Misleading

$\square \square \square \square$ = Not Informative

पढ口 $\square \square$ = Potentially Informative

पढ口๐ $\square$ = Reliable

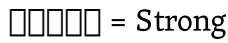

To read the reviews, click the links below. 